\title{
A Multiuser Detector Based Method to Improving GPS Receiver Sensitivity
}

\author{
Siyuan Liang
}

Shaanxi Key Laboratory of Information Communication Network and Security (Xi'an University of Posts and Telecommunications), Xi'an 710121, China.

telestorm@163.com

Keywords: GPS, Receiver Sensitivity, Kalman Filtering, Anti Multi-Access Interference.

\begin{abstract}
The fainter receiving signal power of GPS is a key factor to limit its applications, then the main solution to this problem is improving receiver sensitivity. This paper proposes a Kalman filtering multiuser detector method to anti multi-access interference (MAI) that can improve receiver sensitivity. This method is verified by simulation, the results shows sensitivity improving value about $1 \mathrm{~dB}$.
\end{abstract}

\section{Introduction}

The high-precision and wide coverage lead to the Global Navigation Satellite Systems (GNSS) has been widely used. Currently, the BDS has covered the Asia Pacific region, and then it will achieve global seamless coverage in 2020. In China, the BDS and GPS are mainly used [1].

The arriving satellite signal is very weak is an obvious shortage of GNSS. In downtown environment, the building and trees can interrupt the signal to reduce power, which influences the receiving and positing of GNSS receiver. This reason restricts the GNSS application. It is direct method to solve this problem that improving receiver sensitivity.

BDS and GPS are asynchronous direct sequence code division multiple access (DS-CDMA) system, the satellites are source and the receivers are destination [2]. The receiver needs multiple satellites signal to complete position, which will generates MAI. The MAI can reduce signal to noise ratio (SNR) and deteriorate receiver sensitivity. This paper proposes a method to anti MAI based on Kalman filtering multiuser detector, which can improve receiver sensitivity to solve the problem of fainter signal receiving.

\section{Mathematical Models}

BDS and GPS have used binary phase shift keying (BPSK) modulation and their spreading codes are gold code and C/A code, respectively. The Fig. 1 shows MAI generated process.

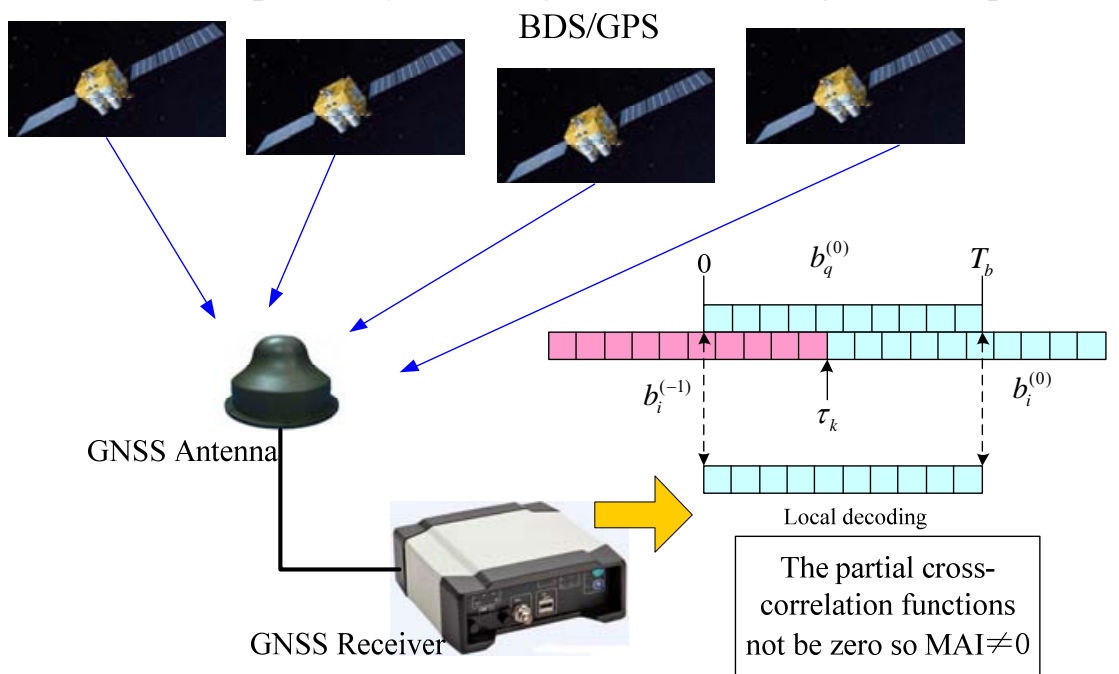

Fig 1. MAI generated process 
The signal at receiver antenna shows by equation (1).

$$
r(t)=\sum_{i=1}^{M} A_{i} b_{i}\left(t-\tau_{i}\right) s_{i}\left(t-\tau_{i}\right) \cos \left(\omega_{c} t+\phi_{i}\right)+n(t)
$$

The $A_{i}$ is signal amplitude of the $i$ satellite, $b_{i}(t) \in\{1,-1\}$ is data of the $i$ satellite, $s_{i}(t)$ is spreading codes of the i satellite. The $\tau_{i}$ is time delay between different satellites, $\omega_{c}$ is frequency of carrier, $\phi_{i}$ is i satellite's phase information, $n(t)$ is the receiver's thermal noise with sided spectral density $N_{0} / 2$. The i satellite signal dispreading process shows by equation (2).

$$
\hat{b}_{q}=N T_{c} A_{q} b_{q}+A_{q} \sum_{\substack{i=1 \\ i \neq q}}^{M}\left[b_{i}^{(-1)} R_{i, q}\left(\tau_{i}\right)+b_{i}^{(0)} \hat{R}_{i, q}\left(\tau_{i}\right)\right]+\int_{0}^{T_{b}} n(t) s_{q}(t) d t
$$

In the equation (2), part I is desired signal q satellite, part II is MAI and part III is thermal noise. The $\mathrm{N}$ and $T_{c}$ are spreading code length and chip width, respectively. In the part II, $\hat{R}_{i, q}\left(\tau_{i}\right)$ and $\hat{R}_{i, q}\left(\tau_{i}\right)$ are partial cross-correlation functions of $\mathrm{q}$ and $\mathrm{i}$ satellite that not be zero in asynchronous DS-CDMA system. In the part III, the thermal noise likes additive white Gauss noise (AWGN) that not be affected by spreading and dispreading.

Defining

$$
\begin{aligned}
s_{i} & =\left[s_{i}(0), s_{i}(1), s_{i}(2) \ldots s_{i}(N-1)\right] \\
y & =[r(0), r(1), r(2) \ldots r(N-1)] \\
v & =[n(0), n(1), n(2) \ldots n(N-1)]
\end{aligned}
$$

After demodulation, the receiving signal express in vector form, as equation (4).

$$
y(k)=\sum_{i=1}^{M} A_{i}(k) b_{i}(k) s_{i}(k)+v(k)
$$
[3]

The multiuser detector for satellite q can be used to anti MAI, the output is given by equation (5).

$$
\hat{b}_{q}(k)=\operatorname{sgn}\left(<c_{q}(k), y(k)>\right)=\operatorname{sgn}\left(c_{q}^{T}(k) y(k)\right)
$$

In the equation (5), the tap-weight vector $c_{q}(k)$ characterizes the multiuser detector. In the following, we consider how to establish $c_{q}(k)$ for blind adaptive multiuser detector. The $c_{q}(k)$ can be expressed as equation (6).

$$
c_{q}(k)=s_{q}-C_{q, \text { null }} \bullet w_{q}(k)
$$

Where, the $C_{q, \text { null }}$ is a $N \times(N-1)$ matrix, that is the null space of $s_{q}$. The $c_{q}(k)$ can adaptively change with weight vector $w_{q}(k)$.

\section{Method Based on Kalman Filtering}

An adaptive multiuser detection based on Kalman Filtering is designed to anti-MAI in BDS/GPS receiver. The state equation and measurement equation are foundations of Kalman Filtering designing, which constitute the dynamic system model of multiuser detection [4].

Defining:

$$
z_{q}(k)=s_{q}^{T} y(k), d^{T}(k)=y^{T}(k) C_{q, n u l l}
$$

If $w_{q}(t)$ achieves $w_{q, o p t}(k)$, the measurement equation as following $(6)$.

$$
z_{q}(k)=d(k) w_{q, o p t}(k)+e_{q}(k)
$$

The Kalman filtering equations as following:

The state estimation equation:

The gain equation:

$$
w_{q, o p t}(k)=w_{q, o p t}(k-1)+g(k)\left[z(k)-d^{T}(k) w_{q, o p t}(k-1)\right]
$$

$$
g(k)=K(k \mid k-1) d(k)\left[d^{T}(k) K(k \mid k-1) d(k)+\xi_{m m}\right]^{-1}
$$

The MSE estimation equation:

$$
K(k+1 \mid k)=K(k \mid k-1)-g(k) d^{T}(k) K(k \mid k-1)
$$

The tap-weight vector equation: 


$$
c_{q}(k)=s_{q}-C_{q, \text { null }} \bullet w_{q, o p t}(k)
$$

Where, $g(k)$ is gain of Kalman filtering, $K(k+1 \mid k)$ is MSE matrix and $K(1 \mid 0)=I$. In equation (10), $\xi_{m m}$ is the minimum mean output energy of the dynamical system of satellite q, we can directly take $\xi_{m m}(k)=A_{q}(k)$.

\section{Simulation and Results}

The method is proved by simulation, the simulation system shows as Fig. 2.

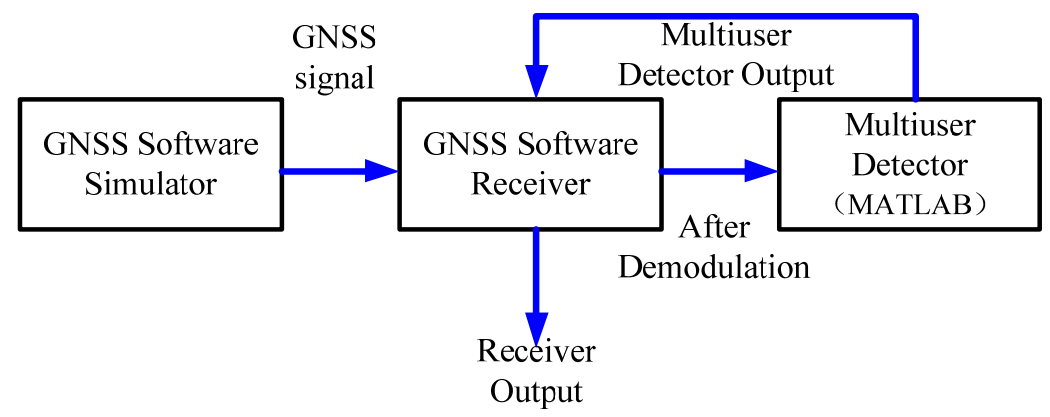

Fig 2. The simulation system

The GNSS software simulator generates BDS/GPS signal and received and processed by software receiver, which includes Kalman filtering multiuser detector. The multiuser detector is realized by MATLAB.

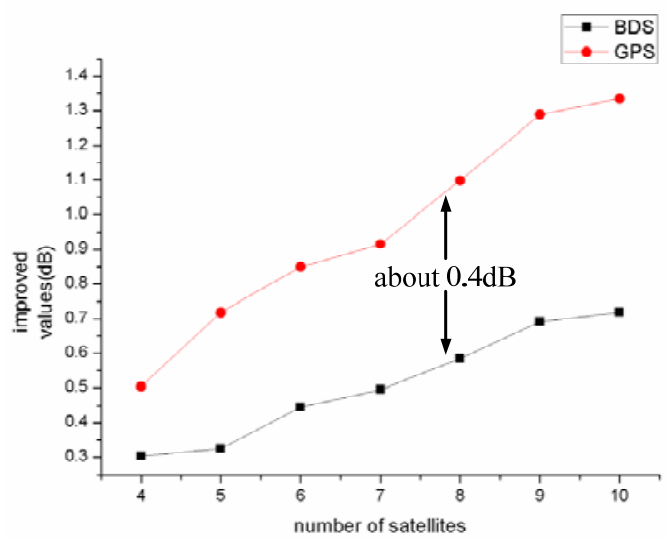

Fig 3. The results of receiver sensitivity improved

The Fig. 3shows the results of receiver sensitivity improved, the horizontal axis indicates the number of receiving satellites and the vertical axis indicates the values of sensitivity improved. The MAI grows with number of receiving satellites. The codes length of GPS and BDS are 1M and2M, respectively [5]. The longer codes length make BDS has a stronger ability to anti-MAI, so the receiver sensitivity improved values of BDS less than GPS and the difference value is about $0.4 \mathrm{~dB}$ based on simulation. The simulation result show, when the GPS receiver receiving more than 8 satellites, the improved value is about $1 \mathrm{~dB}$; when the BDS receiver receiving more than 8 satellites, the improved value is about $0.5 \mathrm{~dB}$.

\section{Conclusion}

The improved sensitivity can take receiver stronger signal acquisition ability to enhance the BDS/GPS usability. In this paper, a Kalman filtering multiuser detector is proposed to anti-MAI that can improve receiver sensitivity. The method availability is proved by simulation results. 


\section{Acknowledgments}

This work was supported by Scientific Research Plan Projects of Shaanxi Education Department (No. 17JK0702) and National Natural Science Foundation of China under Grant 61371087.

\section{References}

[1]. Xin Jie, Zhao Wei, etc. Development of GNSS and Characteristic Analysis of Military Application, Journal of Navigation and Positioning, Vol. 3(2015) No. 11, p. 38-43.

[2]. J. Kouba and P. Héroux, "Precise point positioning using IGS orbit and clock products," GPS solutions, Vol. 5 (2001) No. 2, p. 12-28.

[3]. Wang, G., Lu, Y.: 'Clutter rank of STAP in MIMO radar with waveform diversity', IEEE Trans. Signal Process., 2010, 58, (2), pp. 938-943.

[4]. Y. Wang, C. D. Zhang, X. G. Hu, and Y. Z. Song, et al., "Robust Kalman filter based on different satellite types and its application in GPS/BDS precise point positioning," Journal of Chinese Inertial Technology, Vol. 24 (2016) No. 6, p. 769-779.

[5]. Mao Hu, Wu Dewei, Evaluation of Anti-multipath Performance to Satellite Navigation Signal Tracking on L1/E1/B1 Frequency Band, Journal of System Simulation, Vol. 28(2016) No. 1, p. 106-113. 